\title{
-NOTES-
}

\section{A PARADOX IN MICROCONTINUUM FLUID MECHANICS*}

BY H. RAMKISSOON (University of the West Indies, St. Augustine, Trinidad)

1. Introduction. The main purpose of this work is to reveal a paradox associated with a linearized version of a complex system of coupled differential equations frequently encountered in the recently established field of microcontinuum fluid mechanics. This is an extension of the interesting classical result of Olmstead [1], who showed that for a rather large class of approximations to the Navier-Stokes equations, the drag experienced by any body translating with constant velocity remains invariant under flow reversal regardless of body shape. It will be shown here that for the very same class of approximations, this paradoxical property of the drag holds for so-called micropolar fluids [2]. In addition, the positivity of the drag will be established and integral representations for the velocity vector and the independent microrotation vector will be generated.

2. Reciprocal relationship. A linearized version of the equations of motion characterizing steady flow of a micropolar fluid are given by [2]

$$
\begin{aligned}
t_{j i, j}+f_{i} & =a_{j} u_{i, j}, \\
m_{j i, j}+\varepsilon_{i j k} t_{j k}+l_{i} & =\sigma a_{j} v_{i, j},
\end{aligned}
$$

while the linear constitutive laws take the form

$$
\begin{aligned}
t_{i j}(\mathbf{u}, \mathbf{v}) & =\left(-p+\lambda u_{k, k}\right) \delta_{i j}+\frac{1}{2}(2 \mu+\kappa)\left(u_{i, j}+u_{j, i}\right)+\kappa \varepsilon_{i j k}\left(\omega_{k}-v_{k}\right), \\
m_{i j}(\mathbf{v}) & =\alpha v_{k, k} \delta_{i j}+\beta v_{i, j}+\gamma v_{j, i} .
\end{aligned}
$$

Here $t_{i j}$ are the components of the Cauchy stress tensor, $m_{i j}$ are the components of the couple stress tensor, $\mathbf{u}$ is the velocity vector, $\boldsymbol{v}$ is the microrotation vector, $\mathbf{f}$ is the body force, $\mathbf{l}$ is the body couple, $\mathbf{a}$ is a vector specified $a$ priori, $p$ is the pressure, $\varepsilon_{i j k}$ is the alternating tensor, $\delta_{i j}$ is the Kroneker delta, $(\alpha, \beta, \lambda, \mu, \kappa)$ are material coefficients, $\sigma$ is the gyration parameter and

$$
\omega_{k}=\frac{1}{2}(\nabla \times \mathbf{u})_{k} .
$$

In the case of incompressible flows, the above equations (1) and (2) reduce to the following system of coupled vector differential equations:

$$
\begin{array}{r}
-(\mu+\kappa) \boldsymbol{\nabla} \times \boldsymbol{\nabla} \times \mathbf{u}+\kappa \boldsymbol{\nabla} \times \mathbf{v}-\nabla p-(\mathbf{a} \cdot \boldsymbol{\nabla}) \mathbf{u}+\mathbf{f}=0, \\
(\alpha+\beta+\gamma) \boldsymbol{\nabla} \cdot \boldsymbol{v}-\gamma \boldsymbol{\nabla} \times \boldsymbol{\nabla} \times \boldsymbol{v}+\kappa \boldsymbol{\nabla} \times \mathbf{u}-2 \kappa \boldsymbol{v}-\sigma(\mathbf{a} \cdot \boldsymbol{\nabla}) \mathbf{v}+\mathbf{l}=0, \\
\boldsymbol{\nabla} \cdot \mathbf{u}=0 .
\end{array}
$$

\footnotetext{
* Received November 5, 1979.
} 
The system (3) is a complex set of coupled differential equations which, together with appropriate boundary conditions, determine the unknown field parameters $\mathbf{u}, \boldsymbol{v}$ and $p$. As regards $\mathbf{a}$, it is an approximation to a velocity field and it is therefore not unreasonable for us to make the assumption that

$$
\boldsymbol{\nabla} \cdot \mathbf{a}=0
$$

The particular cases $\mathbf{a}=0$ and $\mathbf{a}=$ constant (non-zero) give rise to a Stokes-type and an Oseen-type approximation, respectively.

The following reciprocal relationship between two different flow configurations will now be proved:

TheOREM 1. Let $\left(\mathbf{u}^{\prime}, \mathbf{v}^{\prime}, \mathbf{a}^{\prime}, p^{\prime}, \mathbf{f}^{\prime}, \mathbf{l}^{\prime}\right)$ and $\left(\mathbf{u}^{\prime \prime}, \mathbf{v}^{\prime \prime}, \mathbf{a}^{\prime \prime}, p^{\prime \prime}, \mathbf{f}^{\prime \prime}, \mathbf{l}^{\prime \prime}\right)$ represent any two motions of the same fluid which conform to Eqs. (1)-(4). If $S$ is a closed surface bounding any fluid volume $V$ and $\mathbf{u}^{\prime}, \mathbf{u}^{\prime \prime}, \mathbf{v}^{\prime}, \mathbf{v}^{\prime \prime} \in C^{1}$ in $S+V$, then

$$
\begin{aligned}
& \int_{S}\left[\left(\mathbf{t}^{\prime} \cdot \mathbf{u}^{\prime \prime}-\mathbf{t}^{\prime \prime} \cdot \mathbf{u}^{\prime}\right)+\left(\mathbf{m}^{\prime} \cdot \mathbf{v}^{\prime \prime}-\mathbf{m}^{\prime \prime} \cdot \mathbf{v}^{\prime}\right)\right] d S \\
& \quad+\int_{V}\left[\left(\mathbf{f}^{\prime} \cdot \mathbf{u}^{\prime \prime}-\mathbf{f}^{\prime \prime} \cdot \mathbf{u}^{\prime}\right)+\left(\mathbf{l}^{\prime} \cdot \mathbf{v}^{\prime \prime}-\mathbf{l}^{\prime \prime} \cdot \mathbf{v}^{\prime}\right)\right] d V \\
& \quad+\int_{V}\left[\left\{\mathbf{u}^{\prime} \cdot\left(\mathbf{a}^{\prime \prime} \cdot \nabla\right) \mathbf{u}^{\prime \prime}-\mathbf{u}^{\prime \prime} \cdot\left(\mathbf{a}^{\prime} \cdot \nabla\right) \mathbf{u}^{\prime}\right\}+\sigma\left\{\mathbf{v}^{\prime} \cdot\left(\mathbf{a}^{\prime \prime} \cdot \nabla\right) \mathbf{v}^{\prime \prime}-\mathbf{v}^{\prime \prime} \cdot\left(\mathbf{a}^{\prime} \cdot \nabla\right) \mathbf{v}^{\prime}\right\}\right] d V=0,
\end{aligned}
$$

where $t_{i}=t_{j i} n_{j}$ with $\mathbf{n}$ being the unit outward drawn normal vector.

Proof. From the equations of motion (1), we have

$$
\begin{aligned}
t_{j i, j}^{\prime}+f_{i}^{\prime} & =a_{j}^{\prime} u_{i, j}^{\prime}, \\
m_{j i, j}^{\prime}+\varepsilon_{i j k} t_{j k}^{\prime}+l_{i}^{\prime} & =\sigma a_{j}^{\prime} v_{i, j}^{\prime} .
\end{aligned}
$$

Multiplying (6) by $u_{i}^{\prime \prime}$ and (7) by $v_{i}^{\prime \prime}$, adding and then integrating the sum over the region $V$, we get with the aid of Gauss' Divergence Theorem

$$
\begin{aligned}
\int_{S}\left(t_{i}^{\prime} u_{i}^{\prime \prime}+m_{i}^{\prime} v_{i}^{\prime \prime}\right) d S+\int_{V}\left(f_{i}^{\prime} u_{i}^{\prime \prime}+l_{i}^{\prime} v_{i}^{\prime \prime}\right) d V-\int_{V}\left(u_{j}^{\prime \prime} a_{i}^{\prime} u_{j, i}^{\prime}\right. & \left.+\sigma v_{j}^{\prime \prime} a_{i}^{\prime} v_{j, i}^{\prime}\right) d V \\
& =\int_{V}\left(t_{j i}^{\prime} u_{i, j}^{\prime \prime}+m_{j i}^{\prime} v_{i, j}^{\prime \prime}-\varepsilon_{i j k} t_{j k}^{\prime} v_{i}^{\prime \prime}\right) d V .
\end{aligned}
$$

Interchanging the primed and double-primed symbols in the foregoing expression and subtracting, we obtain the required reciprocal relationship (5).

It should be noted that in the case of Stokes flows where $\mathbf{a}^{\prime}=\mathbf{a}^{\prime \prime}=0,(5)$ reduces to

$$
\begin{aligned}
\int_{S}\left[\left(\mathbf{t}^{\prime} \cdot \mathbf{u}^{\prime \prime}-\mathbf{t}^{\prime \prime} \cdot \mathbf{u}^{\prime}\right)+\left(\mathbf{m}^{\prime} \cdot \mathbf{v}^{\prime \prime}\right.\right. & \left.\left.-\mathbf{m}^{\prime \prime} \cdot \mathbf{v}^{\prime}\right)\right] d S \\
& +\int_{V}\left[\left(\mathbf{f}^{\prime} \cdot \mathbf{u}^{\prime \prime}-\mathbf{f}^{\prime \prime} \cdot \mathbf{u}^{\prime}\right)+\left(\mathbf{l}^{\prime} \cdot \mathbf{v}^{\prime \prime}-\mathbf{l}^{\prime \prime} \cdot \mathbf{v}^{\prime}\right)\right] d V=0,
\end{aligned}
$$

-a result previously obtained [3]. 
An alternative and sometimes more convenient form of (5) is given by

$$
\begin{aligned}
& 2 \int_{S}\left[\left(\mathbf{t}^{\prime} \cdot \mathbf{u}^{\prime \prime}-\mathbf{t}^{\prime \prime} \cdot \mathbf{u}^{\prime}\right)+\left(\mathbf{m}^{\prime} \cdot \mathbf{v}^{\prime \prime}-\mathbf{m}^{\prime \prime} \cdot \mathbf{v}^{\prime}\right)\right] d S \\
&+2 \int_{V}\left[\left(\mathbf{f}^{\prime} \cdot \mathbf{u}^{\prime \prime}-\mathbf{f}^{\prime \prime} \cdot \mathbf{u}^{\prime}\right)+\left(\mathbf{l}^{\prime} \cdot \mathbf{v}^{\prime \prime}-\mathbf{l}^{\prime \prime} \cdot \mathbf{v}^{\prime}\right)\right] d V \\
&= \int_{V}\left[\mathbf{u}^{\prime \prime} \cdot\left\{\left(\mathbf{a}^{\prime \prime}+\mathbf{a}^{\prime}\right) \cdot \nabla\right\} \mathbf{u}^{\prime}-\mathbf{u}^{\prime} \cdot\left\{\left(\mathbf{a}^{\prime \prime}+\mathbf{a}^{\prime}\right) \cdot \nabla\right\} \mathbf{u}^{\prime \prime}\right] d V \\
&+\int_{V} \sigma\left[\mathbf{v}^{\prime \prime} \cdot\left\{\left(\mathbf{a}^{\prime \prime}+\mathbf{a}^{\prime}\right) \cdot \nabla\right\} \mathbf{v}^{\prime}-\mathbf{v}^{\prime} \cdot\left\{\left(\mathbf{a}^{\prime \prime}+\mathbf{a}^{\prime}\right) \cdot \nabla\right\} \mathbf{v}^{\prime \prime}\right] d V \\
&+\int_{S}\left[\mathbf{u}^{\prime \prime} \cdot \mathbf{u}^{\prime}\left(\mathbf{a}^{\prime}-\mathbf{a}^{\prime \prime}\right) \cdot \mathbf{n}-\sigma \mathbf{v}^{\prime \prime} \cdot \mathbf{v}^{\prime}\left(\mathbf{a}^{\prime}-\mathbf{a}^{\prime \prime}\right) \cdot \mathbf{n}\right] d S .
\end{aligned}
$$

In the absence of body forces and micropolar effects, this reduces to the classical result [1]

$2 \int_{S}\left(\mathbf{t}^{\prime} \cdot \mathbf{u}^{\prime \prime}-\mathbf{t}^{\prime \prime} \cdot \mathbf{u}^{\prime}\right) d S=\int_{V}\left[\mathbf{u}^{\prime \prime} \cdot\left\{\left(\mathbf{a}^{\prime \prime}+\mathbf{a}^{\prime}\right) \cdot \nabla\right\} \mathbf{u}^{\prime}-\mathbf{u}^{\prime} \cdot\left\{\left(\mathbf{a}^{\prime \prime}+\mathbf{a}^{\prime}\right) \cdot \nabla\right\} \mathbf{u}^{\prime \prime}\right] d V$

$$
+\int_{S} \mathbf{u}^{\prime \prime} \cdot \mathbf{u}^{\prime}\left(\mathbf{a}^{\prime}-\mathbf{a}^{\prime \prime}\right) \cdot \mathbf{n} d S
$$

The general reciprocal relationship derived above can be used to obtain integral representations of the unknown parameters of the field equations (3) in terms of so-called fundamental singular solutions. For convenience, we define the body operators $L$ and $M$ as

$$
\begin{aligned}
L(\mathbf{u}, \mathbf{v}, p) & =-(\mu+\kappa) \nabla \times \nabla \times \mathbf{u}+\kappa \boldsymbol{\nabla} \times \mathbf{v}-\nabla p-\mathbf{a} \cdot \nabla \mathbf{u}, \\
M(\mathbf{u}, \mathbf{v}) & =(\alpha+\beta+\gamma) \nabla \nabla \cdot \mathbf{v}-\gamma \nabla \times \nabla \times \mathbf{v}+\kappa \boldsymbol{\nabla} \times \mathbf{u}-2 \kappa \mathbf{v}-\sigma \mathbf{a} \cdot \nabla \mathbf{v},
\end{aligned}
$$

and rewrite (3) as

$$
L(\mathbf{u}, \mathbf{v}, p)=-\mathbf{f}, \quad M(\mathbf{u}, \mathbf{v})=-\mathbf{l}, \quad \nabla \cdot \mathbf{u}=0 .
$$

Let $\mathrm{e}^{k}$ be the unit vector defined along the $k$ th coordinate axis and $\left({ }_{F} u^{k},{ }_{F} v^{k},{ }_{F} p^{k}\right)$ and $\left(c^{u^{k}},{ }_{c} v^{k},{ }_{c} p^{k}\right)$ be the fundamental singular solutions due to a concentrated point force and a concentrated point couple respectively, applied at the point $y$. Thus,

$$
L\left({ }_{F} \mathbf{u}^{k},{ }_{F} v^{k},{ }_{F} p^{k}\right)=-\delta(x-y) \mathbf{e}^{k}, \quad M\left({ }_{F} \mathbf{u}^{k},{ }_{F} v^{k}\right)=0, \quad \nabla \cdot{ }_{F} \mathbf{u}^{k}=0,
$$

and

$$
L\left({ }_{c} \mathbf{u}^{k},{ }_{c} \mathbf{v}^{k},{ }_{c} p^{k}\right)=0, \quad M\left({ }_{c} \mathbf{u}^{k},{ }_{c} \mathbf{v}^{k}\right)=-\delta(x-y) \mathbf{e}^{k}, \quad \nabla \cdot{ }_{c} \mathbf{u}^{k}=0,
$$

for $x \in E$ (infinite space). Here $\delta(x-y)$ is the Dirac delta function and all differentiations are carried out with respect to the variable $x$ with $y$ playing the role of a parameter. 
Applying the reciprocal relationship (5) of Theorem 1 to the systems (10) and (11), we obtain the integral representation for $\mathbf{u}(x)$ as

$$
\begin{aligned}
u_{k}(x)= & \int_{S}\left[\left\{t_{j i}(y)_{F} u_{i}^{k}(x-y)-{ }_{F} t_{j i}^{k}(x-y) u_{i}(y)\right\}\right. \\
& \left.+\left\{m_{j i}(y)_{F} v_{i}^{k}(x-y)-{ }_{F} m_{j i}^{k}(x-y) v_{i}(y)\right\}\right] n_{j} d S \\
& +\int_{V}\left[f_{i}(y)_{F} u_{i}^{k}(x-y)+l_{i}(y)_{F} v_{i}^{k}(x-y)\right] d V \\
& +\int_{V} a_{i}\left[\left\{{ }_{F} u_{i}^{k}(x-y) u_{j, i}(y)-u_{j}(y)_{F} u_{j, i}^{k}(x-y)\right\}\right. \\
& \left.+\sigma\left\{{ }_{F} v_{j}^{k}(x-y) v_{j, i}(y)-v_{j}(y)_{F} v_{j, i}^{k}(x-y)\right\}\right] d V,
\end{aligned}
$$

where ${ }_{F} t_{j i}^{k},{ }_{F} m_{j i}^{k}$ are components of stresses associated with the fundamental solutions. In a similar manner, (10) and (12) lead to an identical representation for $v_{\kappa}(x)$ except that the subscript $F$ is replaced by the subscript $C$.

In the absence of body forces, body couples and couple stresses, (13) reduces to the expected classical result [4]

$$
\begin{aligned}
u_{k}(x)= & \int_{S}\left[t_{j i}(y)_{F} u_{i}^{k}(x-y)-{ }_{F} t_{j i}^{k}(x-y) u_{i}(y)\right] n_{j} d S \\
& +\int_{V} a_{i}\left[{ }_{F} u_{j}^{k}(x-y) u_{j, i}(y)-u_{j}(y){ }_{F} u_{j, i}^{k}(x-y)\right] d V .
\end{aligned}
$$

3. Drag invariance. Our main concern is to establish a drag invariance property and we shall accomplish this with the aid of the reciprocal relationship derived in the last section.

Consider the motion of a rigid body with surface $\Sigma$ which moves with constant translational velocity $-U$ in an infinite micropolar fluid volume $\Omega$ in the absence of body forces and body couples. We shall assume that such a motion is characterized by Eqs. (1)-(4) with $\mathbf{f}=0, \mathbf{l}=0$. Since the velocity field is measured in a frame of reference fixed with respect to the moving obstacle, it is convenient to define

$$
\mathbf{q}=\mathbf{u}-\mathbf{U} .
$$

Hence, from (3) the modified system of field equations governing the flow are now given by

$$
L(\mathbf{q}, \mathbf{v}, p)=0, \quad M(\mathbf{q}, \mathbf{v})=0, \quad \boldsymbol{\nabla} \cdot \mathbf{q}=0 .
$$

As regards boundary conditions, we restrict our attention to those flows which adhere to the exterior of the obstacle and tend to a uniform flow at infinity. Hence

$$
\mathbf{q}=-\mathbf{U}, \quad \mathbf{v}=0, \quad x \in \Sigma
$$

and

$$
\mathbf{q} \rightarrow 0, \quad \mathbf{v} \rightarrow 0, \quad p \rightarrow 0 \quad \text { as } \quad x \rightarrow \infty .
$$


We shall, as done by Olmstead in the classical case [1], require that the linearized velocity a satisfy any one of the boundary conditions

$$
\mathbf{a}=\mathbf{A} \quad \text { on } \Sigma, \quad \mathbf{a} \cdot \mathbf{n}=\mathbf{0} \text { on } \Sigma, \quad \mathbf{a} \rightarrow \mathbf{B} \quad \text { as } \quad x \rightarrow \infty .
$$

Both the Stokes approximation where $\mathbf{a}=0$ and the Oseen approximation where $\mathbf{a}=\mathbf{U}$, satisfy (4) and at least one of the conditions in (17).

As a preliminary result, we shall show that the drag $D(\mathbf{U})$ experienced by the body is positive.

THEOREM 2. Let $\mathbf{q}, \mathbf{v}, p$ associated with the system (14) have a sufficiently rapid asymptotic decay. Then

$$
D(\mathbf{U}) \geq 0 .
$$

Proof. By definition, the drag on the obstacle is given by the integral formula

$$
D(\mathbf{U})=-\frac{\mathbf{U}}{|\mathbf{U}|} \cdot \int_{\Sigma} \mathbf{t} d S .
$$

With the aid of (1), (2) and (4), it can be easily verified that

$$
\int_{S}(\mathbf{t} \cdot \mathbf{q}+\mathbf{m} \cdot \mathbf{v}) d S=\int_{\Omega} H d \Omega+\frac{1}{2} \int_{S}[(\mathbf{q} \cdot \mathbf{q})(\mathbf{a} \cdot \mathbf{n})+\sigma(\mathbf{v} \cdot \mathbf{v})(\mathbf{a} \cdot \mathbf{n})] d S
$$

where the boundary $S$ of the fluid volume $\Omega$ is composed of the body surface $\Sigma$ and the boundary at infinity, and

$$
H=(2 \mu+\kappa) d_{i j} d_{j i}+2 \kappa\left(\omega_{j}-v_{j}\right)\left(\omega_{j}-v_{j}\right)+\alpha v_{j, j} v_{i, i}+\beta v_{j, i} v_{i, j}+\gamma v_{i, j} v_{i, j}
$$

with $d_{i j}=\frac{1}{2}\left(q_{i, j}+q_{j, i}\right)$. Now utilizing the conditions of (15) and (16) in (19), we obtain

$$
-\mathbf{U} \cdot \int_{\Sigma} \mathbf{t} d S=\int_{\Omega} H d \Omega+\frac{1}{2}|\mathbf{U}|^{2} \int_{\Sigma} \mathbf{a} \cdot \mathbf{n} d S .
$$

Under any one of the conditions of (17), the last integral vanishes and hence

$$
D(\mathbf{U})=\frac{1}{|U|} \int_{\Omega} H d \Omega \text {. }
$$

But from thermodynamic considerations [2]

$$
\alpha v_{j, j} v_{i, i}+\beta v_{j, i} v_{i, j}+\gamma v_{i, j} v_{i, j} \geq 0 .
$$

Thus $H \geq 0$ and we obtain the desired result

$$
D(\mathbf{U}) \geq 0 .
$$

The invariance of this drag under flow reversal will now be established.

Definition. A velocity field $\mathbf{V}(x, \mathbf{U})$ is said to be reversible if

$$
\mathbf{V}(x,-\mathbf{U})=-\mathbf{V}(x, \mathbf{U})
$$

at each point $x$ of the flow field.

THEOREM 3. The drag experienced by the rigid translating body remains invariant under the reversal of obstacle velocity if $\mathbf{a}(x, \mathbf{U})$ is reversible. 
Proof. This is a direct application of the reciprocal relationship given by (8). Let (q, $\mathbf{v}$, $p, \mathbf{a}, \mathbf{U})$ and $\left(\mathbf{q}^{\prime}, \mathbf{v}^{\prime}, p^{\prime}, \mathbf{a}^{\prime}, \mathbf{U}^{\prime}\right)$ be any two flow configurations satisfying the system of equations (14) and subject to the boundary conditions (15), (16) and any one in (17). From (8), we get

$$
\begin{aligned}
\mathbf{U} \cdot \int_{\Sigma} \mathbf{t}^{\prime} d S-\mathbf{U}^{\prime} \cdot \int_{\Sigma} \mathbf{t} d S=\frac{1}{2} \int_{\Omega}\left[\mathbf{q}^{\prime} \cdot\left\{\left(\mathbf{a}^{\prime}+\mathbf{a}\right) \cdot \nabla\right\} \mathbf{q}-\mathbf{q} \cdot\left\{\left(\mathbf{a}^{\prime}+\mathbf{a}\right) \cdot \nabla\right\} \mathbf{q}^{\prime}\right. \\
\left.+\sigma \mathbf{v}^{\prime} \cdot\left\{\left(\mathbf{a}^{\prime}+\mathbf{a}\right) \cdot \nabla\right\} \mathbf{v}-\sigma \mathbf{v} \cdot\left\{\left(\mathbf{a}^{\prime}+\mathbf{a}\right) \cdot \nabla\right\} \mathbf{v}^{\prime}\right] d \Omega .
\end{aligned}
$$

Here we have once more assumed a sufficiently rapid decay of the field parameters. Now take the particular case $\mathbf{U}^{\prime}=-\mathbf{U}$. That is, comparison is being made between a given flow for an arbitrary body and the flow generated by the same body moving in the opposite direction with the same speed. Since $\mathbf{a}$ is reversible, $\mathbf{a}^{\prime}+\mathbf{a}=0$. Substitution into (20) yields

$$
D(\mathbf{U})=D(-\mathbf{U}),
$$

which illustrates the paradoxical property of drag indifference to flow reversal.

4. Discussion. A detailed investigation of the paradoxical property of drag invariance under flow reversal has been carried out by Olmstead [1] for the classical NavierStokes theory (the paradox being the lack of dependence on obstacle symmetry). Chester [5] also noted this property for $|\mathbf{U}| \ll 1$ in an earlier paper. In this work we have in fact shown that this indifference of the drag to flow reversal also arises in the linearized micropolar fluid theory. It is a consequence of the reversibility of the linearization velocity $\mathbf{a}(x, \mathbf{U})$. Hence, as in the classical case [1], its validity should be interpreted within the context of slow flows.

\section{REFERENCES}

[1] W. E. Olmstead, Force relationships and integral representations for the viscous hydrodynamical equations, Arch. Rational Mech. Anal. 31, 380-389 (1968)

[2] A. C. Eringen, Theory of micropolar fluids, J. Math. Mech. 16, 1-18 (1966)

[3] H. Ramkissoon and S. R. Majumdar, Representations and fundamental singular solutions in micropolar fluids, Z. Angew. Math. Mech. 56, 197-203 (1976)

[4] I-Dee Chang and R. Finn, On the solutions of a class of equations occurring in continuum mechanics, with application to the Stokes paradox, Arch. Rational Mech. Anal. 7, 388-401 (1961)

[5] W. Chester, On Oseen's approximation, J. Fluid Mech. 13, 557-569 (1962) 